\title{
Drug utilization profile in adult patients with refractory epilepsy at a tertiary referral center
}

\author{
Perfil de utilização de fármacos por pacientes adultos com epilepsia refratária \\ em um centro terciário de referência
}

\author{
Priscila de Freitas-Lima1, André de Oliveira Baldoni², Veriano Alexandre1, Leonardo Regis Leira Pereira², \\ Américo Ceiki Sakamoto'
}

\begin{abstract}
Objective: To evaluate the utilization profile of antiepileptic drugs in a population of adult patients with refractory epilepsy attending a tertiary center. Method: Descriptive analyses of data were obtained from the medical records of 112 patients. Other clinical and demographic characteristics were also registered. Results: Polytherapies with $\geq 3$ antiepileptic drugs were prescribed to $60.7 \%$ of patients. Of the old agents, carbamazepine and clobazam were the most commonly prescribed ( $72.3 \%$ and $58.9 \%$ of the patients, respectively). Among the new agents, lamotrigine was the most commonly prescribed (36.6\% of the patients). At least one old agent was identified in 103 out of the 104 polytherapies, while at least one new agent was prescribed to $70.5 \%$ of the population. The most prevalent combination was carbamazepine + clobazam + lamotrigine. The mean AED load found was 3.3 (range 0.4-7.7). Conclusion: The pattern of use of individual drugs, although consistent with current treatment guidelines, is strongly influenced by the public health system.
\end{abstract}

Keywords: epilepsy, refractory epilepsy, antiepileptic drugs, polytherapy, drug utilization.

\section{RESUMO}

Objetivo: Avaliar o perfil de utilização de fármacos antiepilépticos em uma população de pacientes adultos com epilepsia refratária atendidos em um centro terciário. Método: Análises descritivas dos dados obtidos dos registros médicos de 112 pacientes. Também foram consideradas as características clínicas e demográficas. Resultados: Foram prescritas politerapias com $\geq 3$ antiepilépticos a $60,7 \%$ dos pacientes. Em relação aos fármacos de primeira geração, carbamazepina e clobazam foram os mais frequentemente prescritos (a 72,3\% e $58,9 \%$ dos pacientes, respectivamente). Dentre os novos antiepilépticos, a lamotrigina foi o mais prescrito (36,6\% dos pacientes). Ao menos um antiepiléptico de primeira geração foi encontrado em 103 das 104 politerapias; ao menos um novo antiepiléptico foi prescrito a 70,5\% da população. A combinação mais prevalente foi carbamazepina+clobazan+lamotrigina. A carga média de antiepilépticos foi 3.3 (0.4 a 7.7). Conclusão: O padrão de utilização de antiepilépticos, embora concordante com guias atuais, é fortemente influenciado pelo sistema público de saúde.

Palavras-chave: epilepsia, epilepsia refratária, fármacos antiepilépticos, politerapia, utilização de fármacos.

Approximately one-third of the patients with epilepsy have recurrent seizures in spite of adequate pharmacological treatment and good compliance, characterizing what is known as refractory epilepsy ${ }^{1}$. The lack of control over seizure recurrence has a major impact not only on the patients' clinical and psychosocial status but also on economic consequences that could potentially overload the health care system due to avoidable expenses with regard to medications, hospitalizations, and examinations ${ }^{2}$.
An important portion of the high costs related to the treatment of refractory epilepsy is devoted to the purchase of second-generation antiepileptic drugs (AED), which are more expensive than first-generation agents. It has a special relevance in countries such as Brazil, where the public health care system provides full and free access to the new AED lamotrigine (LTG), vigabatrin (VGB), gabapentin (GBP), and topiramate (TPM) to patients with refractory epilepsy3. Although scarce in Brazil compared to European countries

\footnotetext{
${ }^{1}$ Ribeirão Preto School of Medicine, University of São Paulo, São Paulo SP, Brazil;

${ }^{2}$ Ribeirão Preto Faculty of Pharmaceutical Sciences, University of São Paulo, São Paulo SP, Brazil;

Hospital das Clínicas da Faculdade de Medicina de Ribeirão Preto, Ribeirão Preto SP, Brazil.

Correspondence: Priscila de Freitas-Lima; Hospital das Clínicas da Faculdade de Medicina de Ribeirão Preto; Universidade de São Paulo; Av. dos Bandeirantes 3900 / CIREP - $4^{\circ}$ andar / Bairro Monte Alegre;14048-900 Ribeirão Preto SP - Brasil; E-mail: priscilalima@usp.br / priscilalima_12@hotmail.com Conflict of interest: There is no conflict of interest to declare.

Support: Conselho Nacional de Desenvolvimento Científico e Tecnológico (CNPq) and Coordenação de Aperfeiçoamento de Pessoal de Nível Superior (CAPES). Received 04 June 2013; Accepted 12 June 2013.
} 
and the United States ${ }^{4}$, pharmacoepidemiological studies are valuable tools that contribute to the evaluation of effectiveness and rationality of AED prescriptions and associations ${ }^{5,6}$, which directly reflects on public health policies.

The purpose of the present study was to evaluate the utilization profile of AED in a population of adult patients with refractory epilepsy, attending a tertiary referral center in Brazil. Factors influencing the prescription profiles as well as clinical and demographic peculiarities are also discussed.

\section{METHOD}

Medical records from 112 outpatients in regular treatment at the Hospital das Clínicas da Faculdade de Medicina de Ribeirão Preto (HCFMRP), Brazil, were evaluated and included in a database created using Microsoft Excel for the specific purpose of this cross-sectional survey. Patient selection was based on convenience sampling, and the inclusion criteria were age $\geq 8$ years and established diagnosis of refractory epilepsy, defined as persistence of seizures after adequately applied treatment(s) with one or more appropriate AED at maximally tolerated doses, excluding treatments whereby titration to usually effective dosages was prevented by the onset of idiosyncratic reactions ${ }^{7}$. Age, gender, age at onset of seizures, antiepileptic therapy details, pharmacoresistance grading, concomitant non-AED medications, types of seizure, and epilepsy according to the International League Against Epilepsy (ILAE) classification ${ }^{8,9}$, and spontaneous reports of adverse effects were registered. Evaluation of AED dosages was performed by calculating the ratio of the prescribed daily dose (PDD) over the defined daily dose (DDD) (PDD/DDD) ${ }^{10}$. DDD is a unit of measurement commonly applied in drug utilization researches and is defined as the assumed average maintenance dose per day of a drug used on its main indication in adults ${ }^{11}$.

Data analyses initially consisted of descriptive statistics for demographics, clinical, and drug utilization profiles. Inferential statistics was used to evaluate potential differences in AED load based on the occurrence of adverse effects. Twotailed unpaired Student's t-test and Chi-square test were used, as pertinent. The correlation between the number of AED and the drug load was assessed by Spearman's correlation test. The software used for statistics was the GraphPad InStat, and the level of significance adopted was $p \leq 0.05$. The present study was approved by the Comitê de Ética em Pesquisa of HCFMRP, Brazil.

\section{RESULTS}

\section{Population characteristics}

One hundred and twelve patients were enrolled from May 2010 to August 2010 at the refractory epilepsy clinic of
HCFMRP. This sample size represents approximately $13 \%$ of all adult patients attending the referred center. Demographic details and epilepsy-related characteristics are expressed in Table 1.

The mean age and standard deviation (SD) for the entire sample $(n=112)$ were $38.8 \pm 11.8$ years; the mean age was 38.5 years for men (range 19-64 years) and 39.1 years for women (range 18-69 years). The majority of patients were diagnosed with localization-related epilepsy (108/112). Among the symptomatic cases, the most frequent etiology by far was hippocampal sclerosis (57.8\% of all the symptomatic cases), followed by malformations of cortical development such as dysplasia, neuronal heterotopia, and polymicrogyria $(13.2 \%$ of the patients with symptomatic localization-related epilepsy). Excluding the 16 seizure-free patients, complex partial seizure was the most common seizure type reported (56/96), which is in accordance with the high prevalence of hippocampal sclerosis observed, an etiology known to have partial seizures as its main ictal manifestation ${ }^{12}$. Both, daily and monthly seizures were reported by $25.9 \%$ of the patients.

Most patients (52.7\%) had failed to respond to maximally tolerated doses of three or more AED used sequentially or, more often, in combination, being eventually classified within grade 3 of pharmacoresistance ${ }^{7}$, as seen in Figure 1.

Table 1. Patient demographics and epilepsy-related characteristics $(n=112)$.

\begin{tabular}{lc}
\hline Patient characteristics & Number of patients (\%) \\
\hline Gender distribution & $64 \mathrm{~F}(57.1) ; 48 \mathrm{M}(42.9)$ \\
Age (years \pm SD) & $38.8 \pm 11.8$ \\
Age at onset of seizures (years \pm SD) & $11.1 \pm 11$ \\
Type of epilepsy & \\
Localization related epilepsy & $108(96.4)$ \\
Cryptogenic & $25(22.3)$ \\
Symptomatic & $83(74.1)$ \\
Generalized epilepsy & $3(2.7)$ \\
Idiopathic & $2(1.8)$ \\
Symptomatic & $1(0.9)$ \\
Undetermined & $1(0.9)$ \\
Type of seizure & \\
Complex partial & $56(58.3)$ \\
Simple partial & $5(5.2)$ \\
Secondarily generalized tonic clonic & $16(16.6)$ \\
Absence & $1(1)$ \\
Tonic & $12(12.5)$ \\
Spasms & $2(2)$ \\
Primarily generalized tonic clonic & $3(3)$ \\
Atonic & $1(1)$ \\
Not classified & $1(1)$ \\
Number of AED $\pm S D$ & $2.6 \pm 0.7$ \\
\hline Unless otherwise indicated, values are number of patients with percen- \\
tages shown in brackets. The seizure type classification considers only \\
patients with ongoing recurrent seizures (96/112). AED: antiepileptic drugs; \\
F: female; M: male; SD: standard deviation.
\end{tabular}




\section{Antiepileptic drug therapies}

A total of 12 AED were identified as being currently administered in our patient population. The mean \pm SD number of AED used per patient was 2.6 \pm 0.7 (Table 1), ranging from one to four AED.

The majority of patients (68 out of 112) were on polytherapy with three or more AED (Figure 2). Among these patients, only 6 patients were prescribed a combination of four AED. Carbamazepine (CBZ) was the AED most commonly found in monotherapy, although not in significant number of patients if considering the entire population (6/112); the other two monotherapies used were LTG and valproic acid (VPA).

Both CBZ and clobazam (CLB) were prescribed to more than $50 \%$ of the patients $(72.3 \%$ and $58.9 \%$, respectively). Following these medications, LTG and TPM were also prescribed to a large proportion of patients (36.6\% and 29.4\%, respectively). Second-generation AED were almost exclusively found in polytherapies (except for one patient using LTG in monotherapy). As expected, at least one firstgeneration AED was found in 103 out of the 104 polytherapies prescribed, while at least one second-generation AED was prescribed to $70.5 \%$ of the patients. Figure 3 presents the frequency of prescription of individual AED in monotherapy or polytherapies, based on patient gender. The most prevalent combination in use was CBZ + CLB + LTG (18 patients).

With respect to the AED prescription patterns regarding specific epileptic syndromes, we found that CBZ was the drug most frequently prescribed to localization-related epilepsies (used by $73.1 \%$ of the patients with this diagnosis), followed by CLB (58.3\%) and LTG (37\%). Only four patients were diagnosed with generalized or undetermined epilepsy, and, among these, the only monotherapy prescribed was VPA.

The dosages were calculated as described under the methods section and the results are displayed in Table 2. Based on mean values, seven out of the $12 \mathrm{AED}$ found in this study were prescribed at dosages within $80 \%-120 \%$ of their respective DDD, namely CBZ, phenytoin (PHT), LTG, levetiracetam (LEV), TPM, VPA, and VGB. Considering that LEV and VGB were prescribed to less than five patients each, their data should be analyzed with caution. Clobazam, clonazepam (CNZ), phenobarbital (PB), oxcarbazepine (OXC), and GBP were not prescribed within the $80-120 \%$ interval of their DDD (84\% above, $62.2 \%$ below, $60.5 \%$ above, $102.5 \%$ above, and $33.4 \%$ below their corresponding DDD, respectively). Furthermore, analysis of the results with OXC and
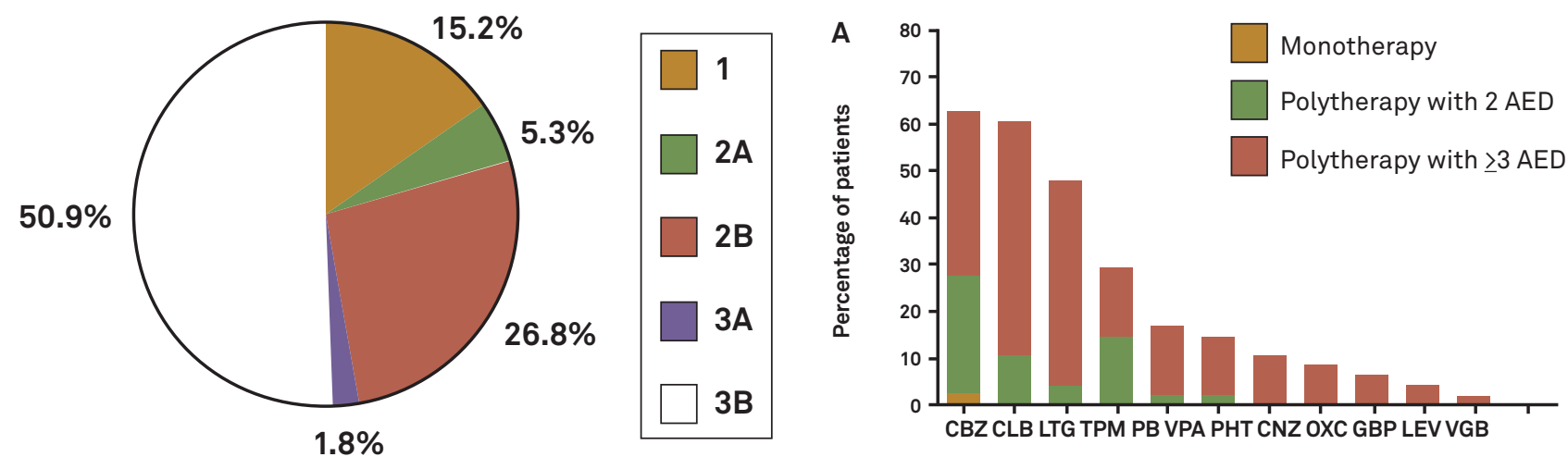

Figure 1. Pharmacoresistance grading. Grade 1: resistant to maximally tolerated dose of one appropriate antiepileptic drug; grade 2: resistant to two AED administered sequentially (2A) or in combination (2B); grade 3: resistant to three AED administered sequentially (3A) or in combination (3B)7.

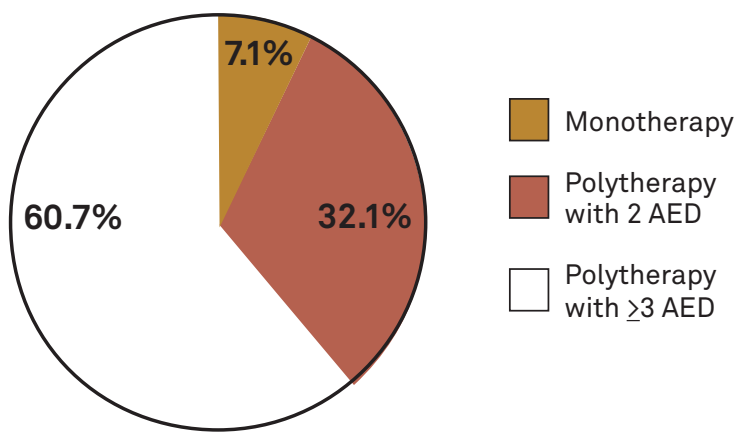

Figure 2. Use of antiepileptic drugs as monotherapy or polytherapy $(n=112)$.

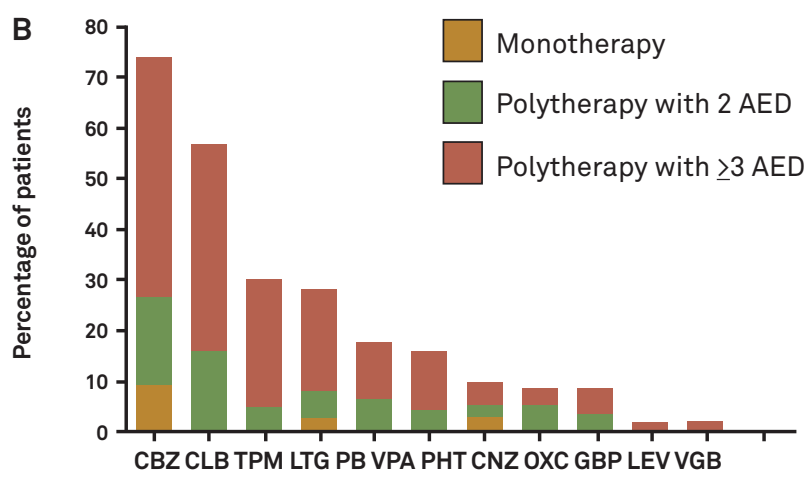

Figure 3. Frequencies of utilization (displayed as percentage of patients) of individual first- and second-generation antiepileptic drugs by men (A) and women (B), either in monotherapy or polytherapies. CBZ: carbamazepine; CLB: clobazam; CNZ: clonazepam; GBP: gabapentin; LEV: levetiracetam; LTG: lamotrigine; OXC: oxcarbazepine; PB: phenobarbital; PHT: phenytoin;TPM: topiramate; VPA: valproic acid;VGB: vigabatrin. 
Table 2. Defined daily doses for each antiepileptic drug and respective dosages (PDD/DDD) in monotherapies and polytherapies (total of 112 patients).

\begin{tabular}{lccc}
\hline Drug & DDD mg/day & $\begin{array}{c}\text { PDD/DDD } \\
\text { monotherapy } \\
\text { mean } \pm \text { SD }(n)\end{array}$ & $\begin{array}{c}\text { PDD/DDD } \\
\text { polytherapy } \\
\text { mean } \pm S D(n)\end{array}$ \\
\hline CBZ & 1000 & $0.93 \pm 0.4(6)$ & $1.11 \pm 0.4(75)$ \\
CLB & 20 & - & $1.8 \pm 1.0(66)$ \\
CNZ & 8 & - & $0.3 \pm 0.1(14)$ \\
PHT & 300 & - & $1.1 \pm 0.3(10)$ \\
PB & 100 & - & $1.6 \pm 0.7(19)$ \\
LTG & 300 & $1.2(1)$ & $1.2 \pm 0.5(40)$ \\
LEV & 1500 & - & $1.2 \pm 0.7(2)$ \\
OXC & 1000 & - & $2.0 \pm 0.6(8)$ \\
TPM & 300 & - & $1.1 \pm 0.6(33)$ \\
VPA & 1500 & $1.0(1)$ & $0.9 \pm 0.4(12)$ \\
GBP & 1800 & - & $0.7 \pm 0.0(2)$ \\
VGB & 2000 & - & $1.0(1)$
\end{tabular}

Dosages (mean $\pm \mathrm{SD}$ ) are expressed as the ratio of prescribed daily dose (PDD) over defined daily dose (DDD). CBZ: carbamazepine; CLB: clobazam; CNZ: clonazepam; GBP: gabapentin; LEV: levetiracetam; LTG: lamotrigine; OXC: oxcarbazepine; PB: phenobarbital; PHT: phenytoin; TPM: topiramate; VPA: valproic acid; VGB: vigabatrin

GBP treatment should take into account that both the drugs were prescribed to only a small number of patients. The restricted number of patients in monotherapy does not allow us to make robust comparisons between the dosages of the AED when used alone or in combination. The AED load was calculated for each patient as the sum of the PDD/DDD ratios for each AED in the treatment regimen. The mean AED load found in our population was 3.3 (range 0.4-7.7). The higher the number of AED prescribed, the higher the AED load, as identified by Spearman's correlation test $(\mathrm{r}=0.63 ; p<0.01)$.

Approximately $76 \%$ of all patients did not spontaneously report the occurrence of any adverse effect, based on an analysis of the medical records. Although the mean AED load in patients with adverse effects did not differ significantly between the patients without adverse effects $(p=0.79)$ and the 27 patients with adverse effects, 17 patients were taking polytherapies with three AED. Dizziness was the most common complaint (37\%), followed by somnolence (22.2\%).

\section{Concomitant use of non-AED medications}

Concomitant non-AED were taken by a total of 41 (36.6\%) patients. Central nervous system (CNS)-related medications were the most commonly found drugs associated with AED; at least one of these agents was taken by 28 of the 41 patients. The most frequently co-prescribed non-AED were, in decreasing order, antidepressants (particularly fluoxetine, sertraline, and amitriptyline), food/mineral supplements (primarily folic acid, used by women), and anxiolytics/hypnotics (particularly diazepam) at the same rank position as antipsychotics (primarily haloperidol).

\section{DISCUSSION}

A tertiary referral center for epilepsy treatment is expected to have a high incidence of cases diagnosed as localizationrelated epilepsy, primarily due to hippocampal sclerosis, an etiology commonly regarded as a difficult-to-treat condition $^{12}$. This clinical characteristic most likely played an important role on the drug utilization profile observed in our population.

In a decreasing order, CBZ, CLB, LTG, and TPM were the most commonly prescribed AED found in the present study, which reasonably agrees with important international guidelines that highlight the efficacy and effectiveness of all available ${ }^{13}$ or only new $\mathrm{AED}^{14}$ for treating partial onset seizures, the most prevalent seizure type in adults ${ }^{15}$. Carbamazepine and PHT are considered by ILAE to have the highest evidence level for use in treating refractory epilepsy as initial monotherapy. Carbamazepine was in fact the most commonly prescribed AED in our population (72.3\%), not only corroborating its therapeutic indication but also in line with the fact that its low cost represents economic advantages when compared to other old, and the new AED. Cost consciousness in health care is a relevant aspect, not only for public health interests but also from the patient's perspective, particularly in developing countries ${ }^{16}$. Although PHT is established as a low-cost effective drug for partial seizures, it has some peculiarities that limit its broad use in clinical context, as observed in the present population. First, PHT has a difficult-to-manage pharmacokinetic profile characterized by variable absorption, high plasma protein binding, and saturable metabolism. Second, this AED exerts important deleterious effects on cognitive functions such as memory, concentration, and mental and motor speed, which, in addition to cosmetic side effects, namely, hirsutism, gengival hyperplasia, and acne, limit its prescription ${ }^{17}$.

The high frequency of CLB prescriptions in our context (58.9\% of all patients), on the other hand, does not follow any international trend. According to Landmark and colleagues ${ }^{18}$, CLB was prescribed to $14 \%$ of their evaluated adult population, similarly to an Italian study $(18 \%)^{19}$. Clobazam was not approved as an AED in the United States by the time the present study was conducted ${ }^{20}$. Nonetheless, for a long time, CLB has been proved to be effective as adjunctive therapy in refractory epilepsy, particularly with predominant partial seizures $^{21}$. In addition, CLB is generally well tolerated and is not a very expensive medication, which favors its prescription as an add-on treatment.

The two other AED importantly found in our study were LTG and TPM. French and colleagues ${ }^{14}$ concluded that all new AED are adequate for use as adjunctive therapy in adults with refractory partial epilepsy. Indeed, there is an international tendency toward increasing prescription of new $\mathrm{AED}^{22}$, sometimes even overcoming the number of prescriptions of 
old $\mathrm{AED}^{18}$. In our study, second-generation $\mathrm{AED}$ were found at least once in $70.5 \%$ of all prescriptions. Topiramate and LTG, similar to most new AED, have a broad efficacy spectrum and favorable pharmacokinetic profile, valuable characteristics particularly when considering co-prescriptions ${ }^{14}$.

It is noteworthy that beside the vast pharmacological arsenal available to treat epilepsy and the support offered for the continuous development of additional treatment options for difficult-to-treat cases $^{23}$, only a limited number of drugs are used in our country, particularly when compared with developed countries such as Norway ${ }^{18}$, Italy ${ }^{19}$, and Denmark ${ }^{22}$. The frequent presence of LEV in international studies and the less important but ever present contributions of drugs such as pregabalin (PGB), tiagabine (TGB), zonisamide (ZNS), and OXC raise the issue of limited access to these drugs due to nation-specific aspects such as availability and $\operatorname{cost}^{24}$. Of these four drugs, only OXC was approved for commercial use in the public health care system of Brazil at the time of data collection, although it was not available free of cost.

In general it has been acknowledged that new AED are equivalent to old agents in terms of efficacy; however, their favorable pharmacokinetic profile is an essential characteristic if we consider that these drugs are initially approved as polytherapies ${ }^{14}$. A second-generation AED constituted an important part of the most prevalent polytherapy in the present study $(\mathrm{CBZ}+\mathrm{CLB}+\mathrm{LTG})$. The rational behind this association may be questioned by some authors because of the similar mechanisms of action of the agents involved; however, the existing national protocol regulating the utilization of new AED (VGB, TPM, LTG, GBP) in cases of refractory epilepsy treated in the Brazilian public health care system claims that only patients who have not clinically answered to the use of at least two monotherapies and one polytherapy can benefit from free access to these $\mathrm{AED}^{3}$. The easier access and the synergism of the combination of CBZ + CLB to treat the prevalent cases of localization-related epilepsy concurred with the protocol requirement for future combinations with a new AED (in case these two agents alone could not achieve seizure control ). Thus, only specific cases will use a combination of one new and one old AED in the context of public health system in Brazil.

The high prevalence of complex polytherapies, though expected, is a recurrent point of concern in the literature. Contradictory to the findings of Malerba and colleagues ${ }^{19}$, not more than four AED were simultaneously prescribed to any of our patients. It should be noted that, as pointed out by Landmark and colleagues ${ }^{18}$ in their study, the total number of polytherapies may be overestimated due to therapy adjustments; sometime two drugs are simultaneously administered, while one is being titrated and the other is still in the process of withdrawal. Even on this convergent scenario of high prevalence of polytherapies among different studies, it is important to consider that there are no evidences that polytherapies are better than monotherapies in achieving seizure control, based on randomized controlled trials ${ }^{25}$.

The occurrence of adverse effects was spontaneously reported only by $24.1 \%$ of our cohort, which partially conflicts with the high prevalence of complex polytherapies and their high mean AED load (3.3; range 0.4-7.7). Such situations are frequently associated with the potential occurrence of pharmacological interactions reflected as signs of adverse effects and/or therapeutic inefficacy and the possible increase in seizure frequency and/or severity as a consequence of overtreatment ${ }^{26,27}$. The relatively low percentage of patients reporting the occurrence of adverse events may be justified by the method of evaluation adopted in the present study. This type of search may underestimate the adverse event of AED compared to investigations based on structured questionnaires ${ }^{27}$. Although not specific and exclusive to benzodiazepines, the high prevalence of dizziness and somnolence as adverse effects may be related to the frequent use of these therapeutic agents. Importantly, we found that clobazam was being used in our population at a mean PDD $84 \%$ above its DDD.

With respect to the use of non-antiepileptic medications, the high prevalence of the use of CNS-related drugs corroborates the findings in Norwegian and Italian patients ${ }^{18,19}$ and the high incidence of psychiatric comorbidities in patients with epilepsy described elsewhere ${ }^{28}$. This scenario usually requires special medical care, since most of the AED can influence the occurrence/aggravation of psychiatric symptoms; in addition, some psychotropic drugs can potentially deteriorate seizure control due to interactions with AED and/or due to their potential epileptogenic effect ${ }^{29}$.

A relevant consideration refers to the lack of difference in AED utilization profile between men and women. Although the AED load was significantly different $(p=0.01)$ between men (3.8 \pm 1.4$)$ and women (3.0 \pm 1.6$)$, the total number of AED was virtually the same in both genders, such that the frequencies of utilization of specific medications were independent of use as monotherapy or polytherapy (data not shown). Considering that $67.2 \%$ of our female population was of reproductive age (up to 44 years), the absence of these differences might at first raise a question if we are treating women the same way we treat men. According to Landmark and colleagues ${ }^{18}$ and Malerba and colleagues ${ }^{19}$, it would be expected that more women than men would use second-generation AED, since these drugs have a low teratogenic potential and risk of interaction with oral contraceptives. To the contrary, the only difference found in our cohort was a higher trend of use of lamotrigine in men compared to women (47.9\% vs. 28.1\%, $p=0.03$ ), which highlights that the main concern in our center is the pursuit of seizure control considering our national limitations.

The discussion around the pharmacological treatment profile in epilepsy is an important point of concern for 
different research groups in different clinical and sociodemographic contexts ${ }^{18,19,22,30}$. To the best of our knowledge, however, there are no Brazilian studies aimed at evaluating the adult population with refractory epilepsy in such a broad clinical approach. It is important to highlight that the current study, although useful, does not represent the adult population with epilepsy as a whole, since the selected sample is composed of drug-resistant cases, a portion known to be around one-third of all patients with epilepsy ${ }^{1}$, and these patients, beside belonging to the wealthiest region of the country, are considered to be a low-income population by the Brazilian public health care system. Thus these patients have restricted access to new AED, driven by political and economic barriers.

In conclusion, our survey provides previously unpublished information on the prescription of AED for adult patients with refractory epilepsy in Brazil. The pattern of use of individual drugs, although consistent with current treatment guidelines, is strongly influenced by our public health care system.

\section{References}

1. Regesta G, Tanganelli P. Clinical aspects and biological bases of drugresistant epilepsies. Epilepsy Res 1999;34:109-122.

2. Tetto A, Manzoni P, Millul A, et al; Osservatorio Regionale per l'Epilessia (OREp). The costs of epilepsy in Italy: a prospective costof-illness study in referral patients with disease of different severity. Epilepsy Res 2002;48:207-216.

3. Brasil, Secretaria de Atenção à Saúde. Portaria SAS/MS nº 864, de 05 de novembro de 2002. Protocolo clínico e diretrizes terapêuticas para epilepsia refratária. Diário Oficial da União, Brasília, DF, 05 nov. 2002.

4. Baldoni AO, Guidoni CM, Pereira LRL. A farmacoepidemiologia no Brasil: estado da arte da produção científica. Rev Univ Vale do Rio Verde 2011:9:78-88.

5. Abasolo-Osinaga E, Abecia-Inchaurregui LC, Etxeandia-Ikobaltzeta I, Burgos-Alonso N, García-del Pozo J. Estudio farmacoepidemiológico del consumo de fármacos antiepilépticos (1992-2004). Rev Neurol 2008:46:449-453

6. Brodie MJ, Sills GJ. Combining antiepileptic drugs - rational polytherapy? Seizure 2011;20:369-375.

7. Perucca E. Pharmacoresistance in epilepsy: how should it be defined? CNS Drugs 1998;10:171-179.

8. Commission on Classification and Terminology of the International League Against Epilepsy. Proposal for revised clinical and electroencephalographic classification of epileptic seizures. Epilepsia 1981;22:489-501.

9. Commission on Classification and Terminology of the International League Against Epilepsy. Proposal for revised classification of epilepsies and epileptic syndromes. Epilepsia 1989;30:389-399.

10. Lammers MW, Hekster YA, Keyser A, Meinardi H, Renier WO, van Lier $\mathrm{H}$. Monotherapy or polytherapy for epilepsy revisited: a quantitative assessment. Epilepsia 1995;36:440-446.

11. World Health Organization Collaborating Centre for Drug Statistics Methodology, Guidelines for ATC classification and DDD assignment, 2012. Available at http://www.whocc.no/atc_ddd_publications/guidelines/

12. Engel Jr J. Introduction to temporal lobe epilepsy. Epilepsy Res 1996;26:141-150.

13. Glauser T, Ben-Menachem E, Bourgeois B, et al. ILAE treatment guidelines: evidence based analysis of antiepileptic drug efficacy and effectiveness as initial monotherapy for epileptic seizures and syndromes. Epilepsia 2006;47:1094-1120.

14. French JA, Kanner AM, Bautista J, et al. Efficacy and tolerability of the new antiepileptic drugs. II. Treatment of refractory epilepsy: report of the TTA and QSS Subcommittees of the American Academy of Neurology and the American Epilepsy Society. Epilepsia 2004;45:410-423. Erratum in: Epilepsia 2004:45:1299.
15. Perucca E, Tomson T. The pharmacological treatment of epilepsy in adults. Lancet Neurol 2011;10:446-456.

16. Betting LE, Kobayashi E, Montenegro MA, et al. Tratamento da epilepsia - Consenso dos especialistas brasileiros. Arq Neuropsiquiatr 2003;61:1045-1070.

17. Thompson P, Huppert FA, Trimble M. Phenytoin and cognitive function: Effects on normal volunteers and implications for epilepsy. Br J Clin Psychol 1981;20:155-162.

18. Landmark CJ, Rytter E, Johannessen SI. Clinical use of antiepileptic drugs at a referral centre for epilepsy. Seizure 2007;16:356-364.

19. Malerba A, Ciampa C, De Fazio S, et al. Patterns of prescription of antiepileptic drugs in patients with refractory epilepsy at tertiary referral centres in Italy. Epilepsy Res 2010;91:273-282.

20. Food and Drug Administration 2011 - Available at: http://www accessdata.fda.gov/drugsatfda_docs/label/2011/202067s000lbl.pdf

21. Allen JW, Oxley J, Robertson MM, Trimble MR, Richens A, Jawad SS. Clobazam as adjunctive treatment in refractory epilepsy. Br Med $\mathrm{J}$ 1983;286:1246-1247.

22. Tsiropoulos B, Gichangi A, Andersen M, Bjerrun L, Gaist D, Hallas J. Trends in utilization of antiepileptic drugs in Denmark. Acta Neurol. Scand 2006;113:405-411.

23. Brodie M. Do we need any more new antiepileptic drugs? Epilepsy Res 2001;45:3-6.

24. Noronha ALA, Borges MA, Marques LHN, et al. Prevalence and Pattern of Epilepsy Treatment in Different Socioeconomic Classes in Brazil. Epilepsia 2007; 48:880-885

25. Beghi E, Gatti G, Tonini C, et al.; on behalf on the BASE Study Group. Adjunctive therapy versus alternative monotherapy in patients with partial epilepsy failing on a single drug: a multicentre, randomized, pragmatic, controlled trial. Epilepsy Res 2003:57:1-13.

26. Perucca E, Kwan P. Overtreatment in epilepsy: How does it occur and how it can be avoided. CNS Drugs 2005;19: 897-908.

27. Alexandre $\mathrm{V} J \mathrm{~J}$, Monteiro EA, Freitas-Lima $\mathrm{P}$, et al. Addressing overtreatment in patients with refractory epilepsy at a tertiary referral centre in Brazil. Epileptic Disord 2011;13:56-60.

28. Kanner AM. The use of psychotropic drugs in epilepsy: what every neurologist should know. Sem Neurol 2008;28:379-388.

29. Ruiz-Giménez J, Sánchez-Álvarez JC, Cañadillas-Hidalgo F, SerranoCastro PJ. Antiepileptic treatment in patients with epilepsy and other comorbidities. Seizure 2010;19:375-382.

30. Oun A, Haldre S, Magi M. Use of antiepileptic drugs in Estonia: an epidemiologic study of adult epilepsy. Eur J Neurol 2006;13:465-470. 